\title{
Theoretical Study of 8-hydroxyquinoline Derivatives as Potential Antennas in Lanthanide complexes: Photophysical Properties and Elucidation of Energy Transfer Pathways.
}

\author{
Juan Julian Santoyo-Flores ${ }^{1}$ and Dayan Paez-Hernandez ${ }^{1}$ \\ ${ }^{1}$ Universidad Andres Bello
}

October 22, 2021

\begin{abstract}
A series of 8-hydroxyquinoline derivatives were characterized and tested as potential antennas in a set of designed lanthanide complexes. The molecular structure and ligand localized nature of the excited states were studied in the framework of the multiconfigurational methods CASSCF/NEVPT2 combined with TDDFT- based approaches, which allows applying a fragmentation scheme in the analysis of the most probable sensitization pathway via antenna effect. The photophysical properties of all the complexes and antennas were carefully analyzed, and the most probable energy transfer pathways were elucidated. Rate constants for photophysical processes involved in the mechanism were calculated, showing a significant contribution of the vibronic coupling in all cases and the predominant intersystem-crossing between S1 and T1 states was demonstrated from the analysis of the nature of the wave function of those states. The energy transfer process described herein demonstrates the possibility of $\mathrm{Eu}(\mathrm{III})$ and $\mathrm{Nd}(\mathrm{III})$ sensitization by the studied ligands. The proposed methodology gives a complete picture of the antenna excited state dynamics.
\end{abstract}

\section{Hosted file}

manuscript.pdf available at https://authorea.com/users/442337/articles/542626-theoreticalstudy-of-8-hydroxyquinoline-derivatives-as-potential-antennas-in-lanthanide-complexesphotophysical-properties-and-elucidation-of-energy-transfer-pathways

\section{Hosted file}

main.tex available at https://authorea.com/users/442337/articles/542626-theoreticalstudy-of-8-hydroxyquinoline-derivatives-as-potential-antennas-in-lanthanide-complexesphotophysical-properties-and-elucidation-of-energy-transfer-pathways 

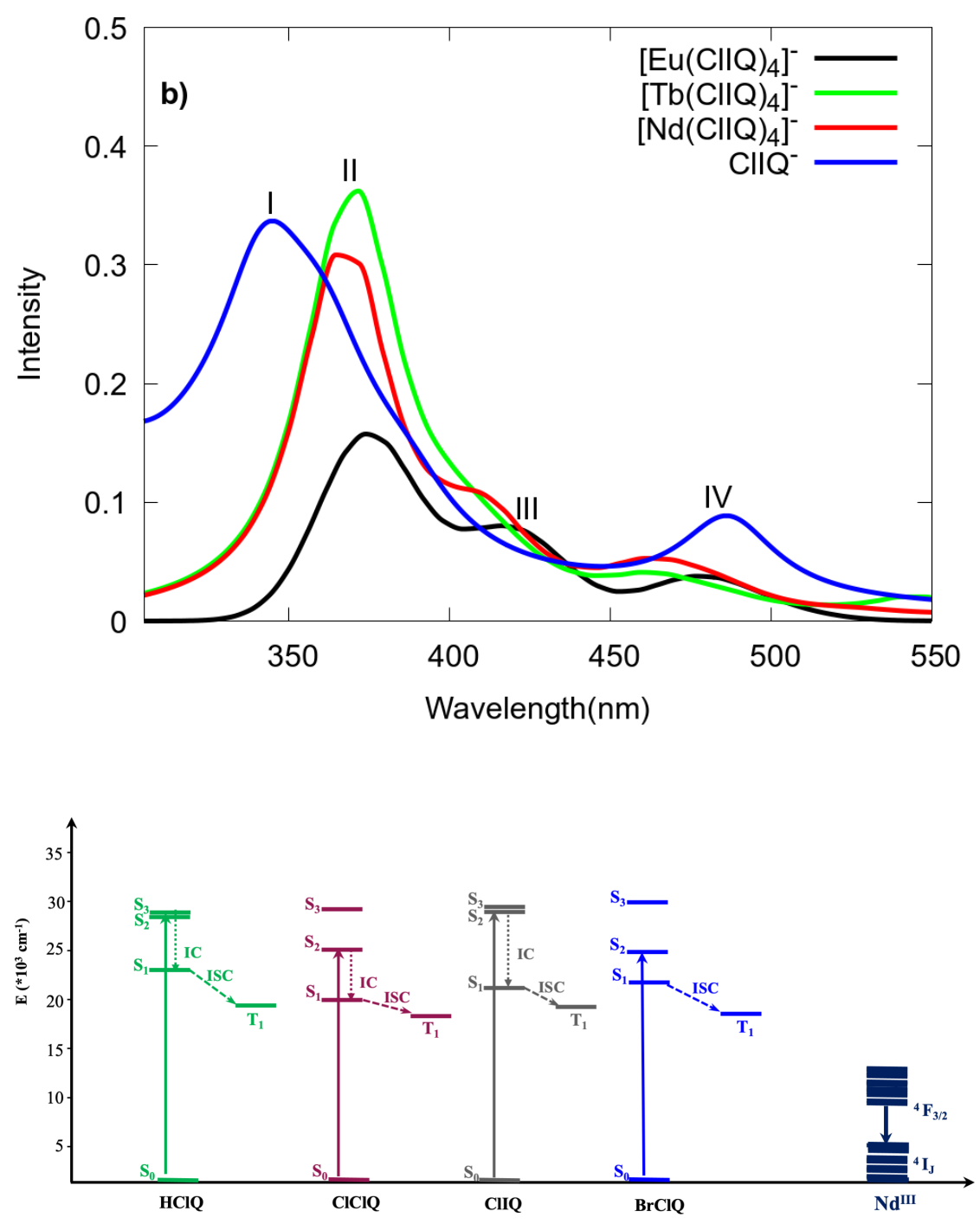

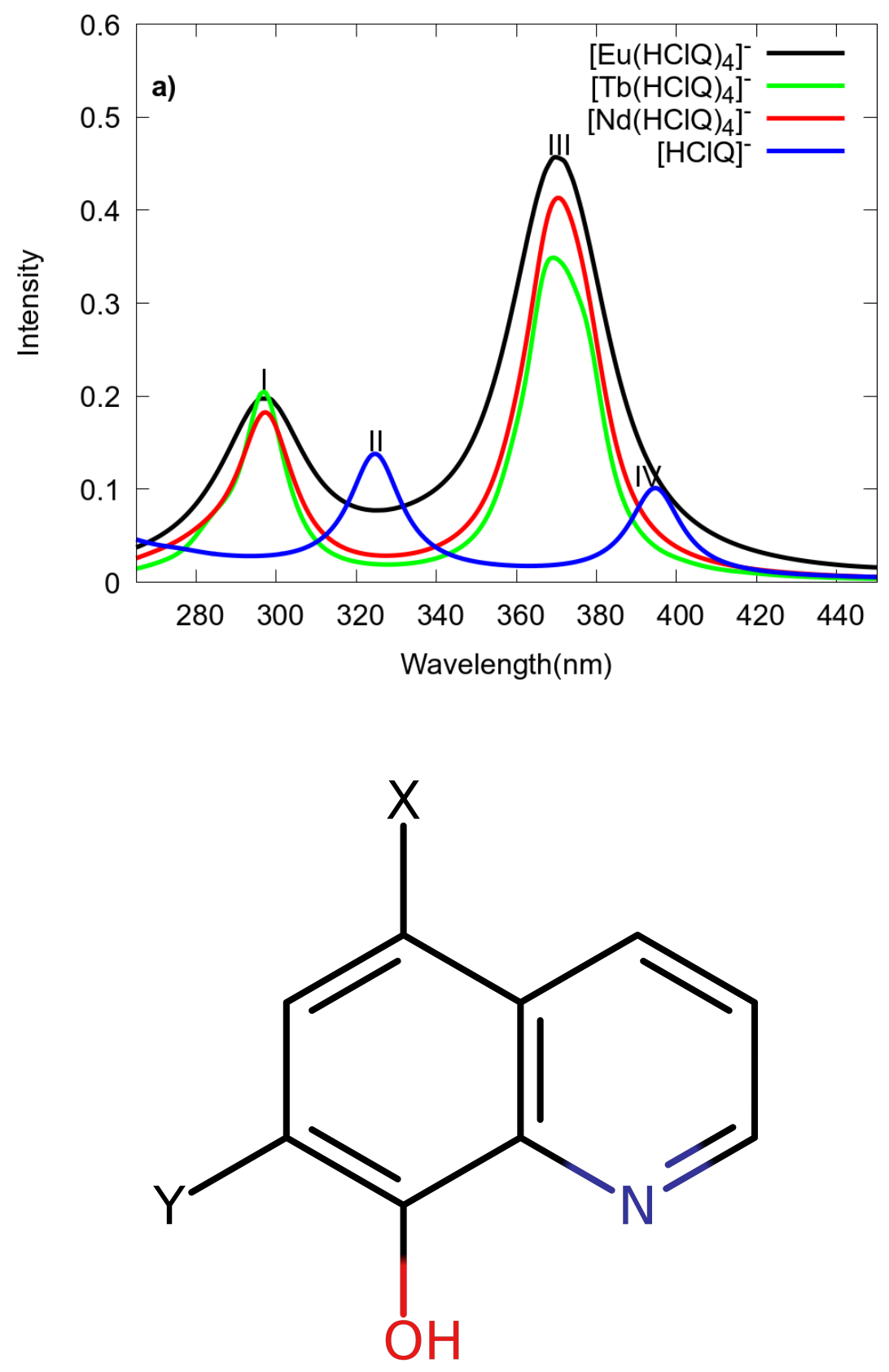

1) $X=\mathrm{Cl}, Y=\mathrm{H}$

2) $X=\mathrm{Cl}, Y=\mathrm{Br}$

3) $X=C l, Y=C l$

4) $X=C l, Y=I$ 


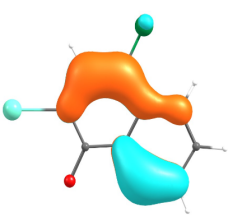

$\pi_{1}(1.831)$

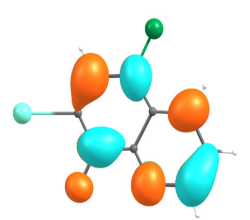

$\pi_{4}(0.678)$

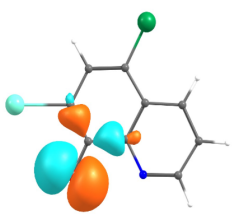

$\mathrm{n}(1.687)$

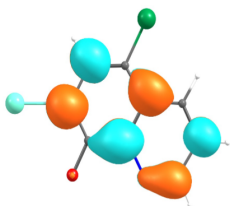

$\pi_{5}(0.448)$

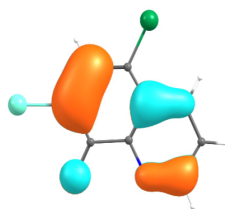

$\pi_{2}(1.599)$

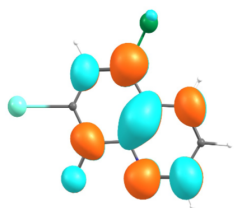

$\pi_{6}(0.292)$

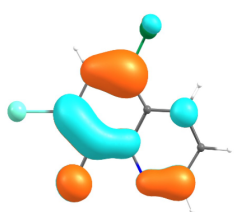

$\pi_{3}(1.338)$

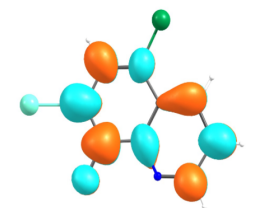

$\pi_{7}(0.125)$

$S_{0} \quad 88 \%\left|\pi_{1}^{2} \pi_{2}^{2} n^{2} \pi_{3}^{2}\right|$

$S_{1} \quad 80 \%\left|\pi_{1}^{2} \pi_{2}^{2} n^{1} \pi_{3}^{2} \pi_{4}^{1}\right|$

$\left(n \rightarrow \pi^{*}\right)$

$T_{1} \quad 75 \%\left|\pi_{1}^{2} \pi_{2}^{2} n^{2} \pi_{3}^{1} \pi_{4}^{1}\right|+10 \%\left|\pi_{1}^{2} \pi_{2}^{2} n^{2} \pi_{3}^{1} \pi_{5}^{1}\right| \quad\left(\pi \rightarrow \pi^{*}\right)$

$T_{2} \quad 81 \%\left|\pi_{1}^{2} \pi_{2}^{2} n^{1} \pi_{3}^{2} \pi_{4}^{1}\right|$

$\left(n \rightarrow \pi^{*}\right)$

$T_{3} \quad 68 \%\left|\pi_{1}^{2} \pi_{2}^{2} n^{2} \pi_{3}^{1} \pi_{5}^{1}\right|+12 \%\left|\pi_{1}^{2} \pi_{2}^{2} n^{2} \pi_{3}^{1} \pi_{4}^{1}\right| \quad\left(\pi \rightarrow \pi^{*}\right)$
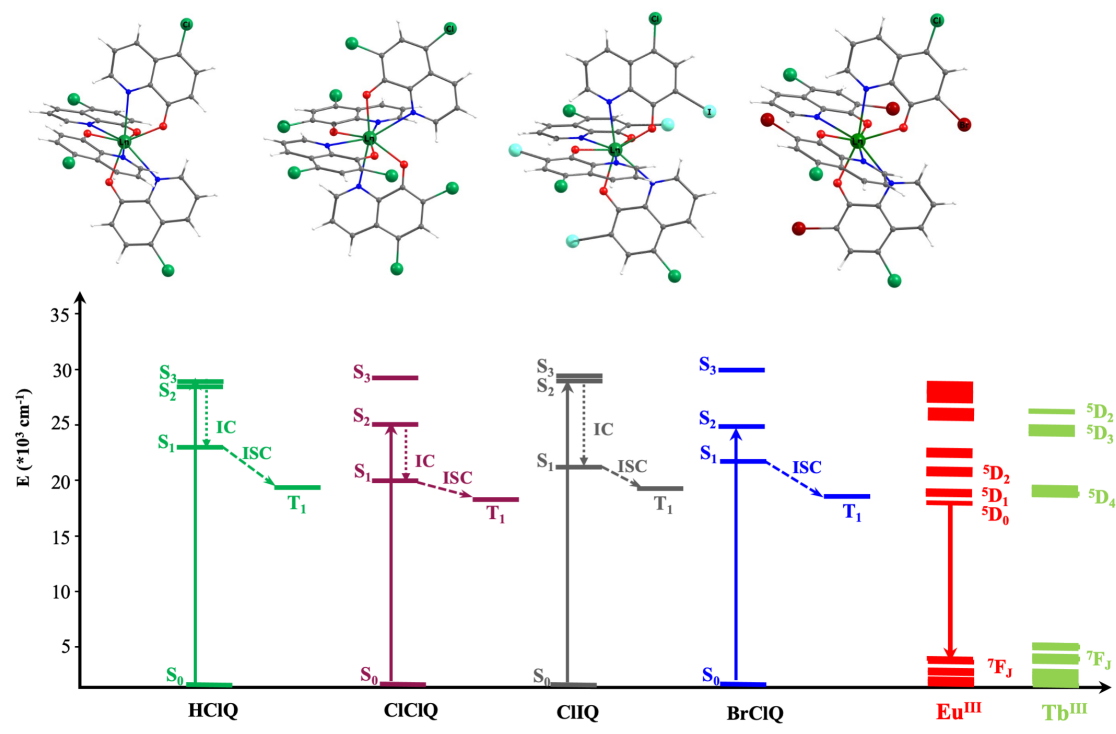


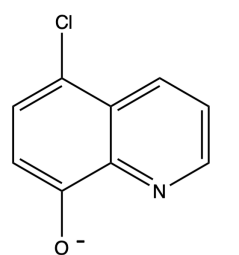

$\mathrm{k}_{\text {fluor }}=1.12 * 10^{7} \mathrm{~s}^{-1}$

$\mathrm{k}_{\mathrm{isc}}=6.50^{*} 10^{7} \mathrm{~s}^{-1}$

$\mathrm{k}_{\text {phos }}=1.22 \mathrm{~s}^{-1}$

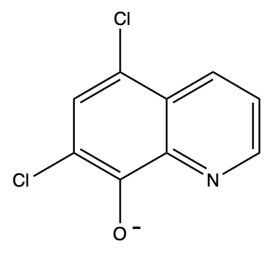

$\mathrm{k}_{\text {fluor }}=7.49 * 10^{6} \mathrm{~s}^{-1}$

$\mathrm{k}_{\mathrm{isc}}=2.94 * 10^{7} \mathrm{~s}^{-1}$

$\mathrm{k}_{\mathrm{phos}}=7.15 \mathrm{~s}^{-1}$

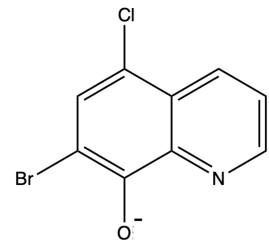

$\mathrm{k}_{\text {fluor }}=8.71 * 10^{6} \mathrm{~s}^{-1}$

$\mathrm{k}_{\text {isc }}=8.46^{*} 10^{7} \mathrm{~s}^{-1}$

$\mathrm{k}_{\text {phos }}=1.10^{*} 10^{2} \mathrm{~s}^{-1}$

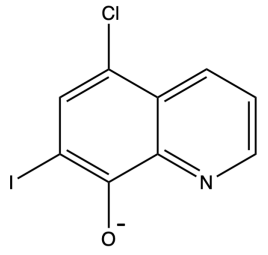

$\mathrm{k}_{\text {fluor }}=7.89 * 10^{6} \mathrm{~s}^{-1}$

$\mathrm{k}_{\mathrm{isc}}=5.78^{*} 10^{8} \mathrm{~s}^{-1}$

$\mathrm{k}_{\text {phos }}=2.70^{*} 10^{2} \mathrm{~s}^{-1}$ 